\title{
The diagnostic accuracy of endobronchial ultrasound and spiral chest computed tomography scan in the prediction of infiltrating and non-infiltrating lymph nodes in patients undergoing endobronchial ultrasound
}

\author{
Sepideh Haghi ${ }^{A, B, C, C, D, E, F}$, Shahram Kahkouee ${ }^{2 A, B, E}$, Arda Kiani ${ }^{3 A, B, D, F}$, Atefeh Abedini ${ }^{A A, B, E, F}$, Neda Akhoundi ${ }^{1 A, B, E, F}$, \\ Mehdi Javanbakht ${ }^{4 A, B, E, F}$, Hadi Rezaei ${ }^{1 A, B, E, F}$, Mersede Paraham ${ }^{1 A, B, F}$ \\ 'College of Medicine, Shahid Beheshti University of Medical Sciences, Iran \\ 2Department of Radiology, Masih Daneshvari Hospital, Shahid Beheshti University of Medical Sciences, Iran \\ ${ }^{3}$ Department of Pulmonary Medicine, Medical University of Shahid Beheshti, Iran \\ 4Economics Unit, Device Access UK Ltd., University of Southampton Science Park, Chilworth, Southampton, United Kingdom
}

\section{Abstract}

\begin{abstract}
Purpose: Endobronchial ultrasound (EBUS) and spiral chest computed tomography (CT) scan are important methods in the prediction of infiltrating and non-infiltrating lymph nodes, and the determination of their diagnostic accuracy would result in a reduction of the burden of problems and an improvement in prognosis. The purpose in this study was to determine the diagnostic accuracy of endobronchial ultrasound and spiral chest CT scan in the prediction of infiltrating and non-infiltrating lymph nodes in patients undergoing endobronchial ultrasound.

Material and methods: In this observational prospective study, 40 consecutive patients with infiltrating and non-infiltrating lymph nodes in Masih-Daneshvari Hospital in 2017 and 2018 were enrolled, and the sensitivity, specificity, and accuracy of EBUS and CT-scan versus fine needle aspiration pathology results were determined in them.

Results: The results in this study demonstrated that the congruence between EBUS and CT scan was $80.5 \%$ $(p=0.0001)$. The sensitivity, specificity, and accuracy for CT scan were $100 \%, 22.6 \%$, and $40 \%$, respectively, and the sensitivity, specificity, and accuracy for EBUS were $100 \%, 16.1 \%$, and $35 \%$, respectively.

Conclusion: According to the obtained results, it may be concluded that CT scan and EBUS results have good congruence and high sensitivity to differentiate infiltrating and non-infiltrating lymph nodes. Hence, these methods are useful for screening methods, but due to their low specificity and accuracy the use of them for a confirmative approach is not beneficial. However, regarding the accessibility and less invasive nature, use of chest CT scan is more rational and is recommended in these patients.
\end{abstract}

Key words: CT scan, lymphadenopathy, EBUS.

\section{Introduction}

Mediastinal and hilar lymphadenopathy is usually diagnosed by computerised tomography (CT) scan, which is also used for staging, with accuracy and specificity of 57\% and $82 \%$, respectively [1]. In surgical staging approximately $18 \%$ of patients with a negative CT scan develop metastatic mediastinal disease [2]. However, all lymph nodes may not be detected. In these cases, usually thoracoscopy

Correspondence address:

Sepideh Haghi, College of Medicine, Shahid Beheshti University of Medical Sciences, Iran, e-mail: hdirezaei@yahoo.com

Authors' contribution:

A Study design · B Data collection · C Statistical analysis · D Data interpretation · E Manuscript preparation · F Literature search · G Funds collection 


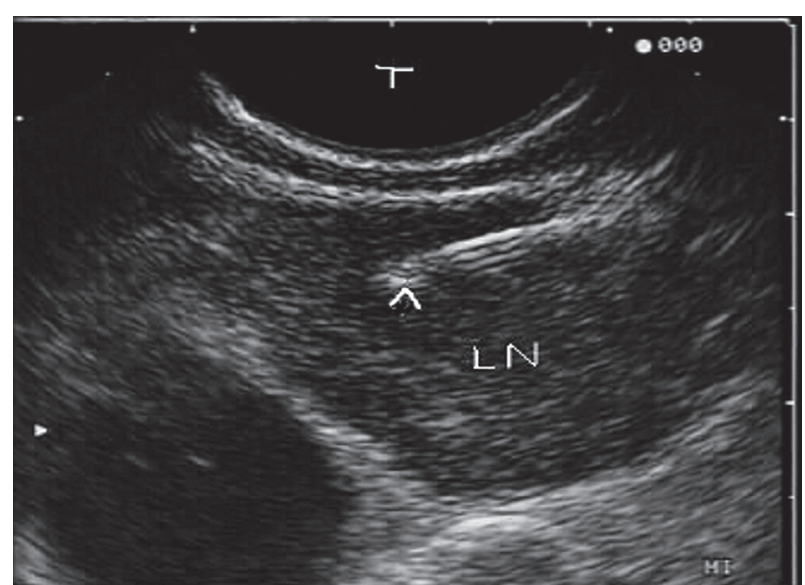

Figure 1. Endobronchial ultrasound fine needle aspiration paratracheal lymph node

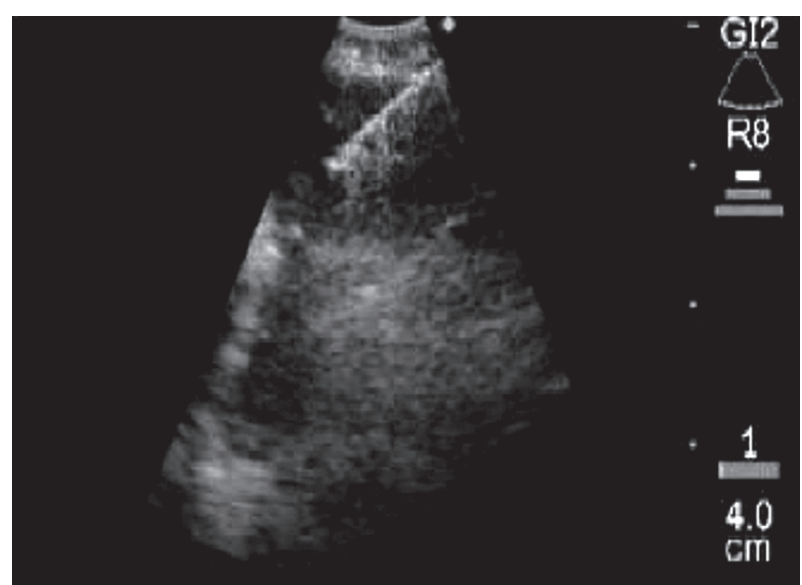

Figure 2. Endobronchial ultrasound fine needle aspiration hilar lymph node

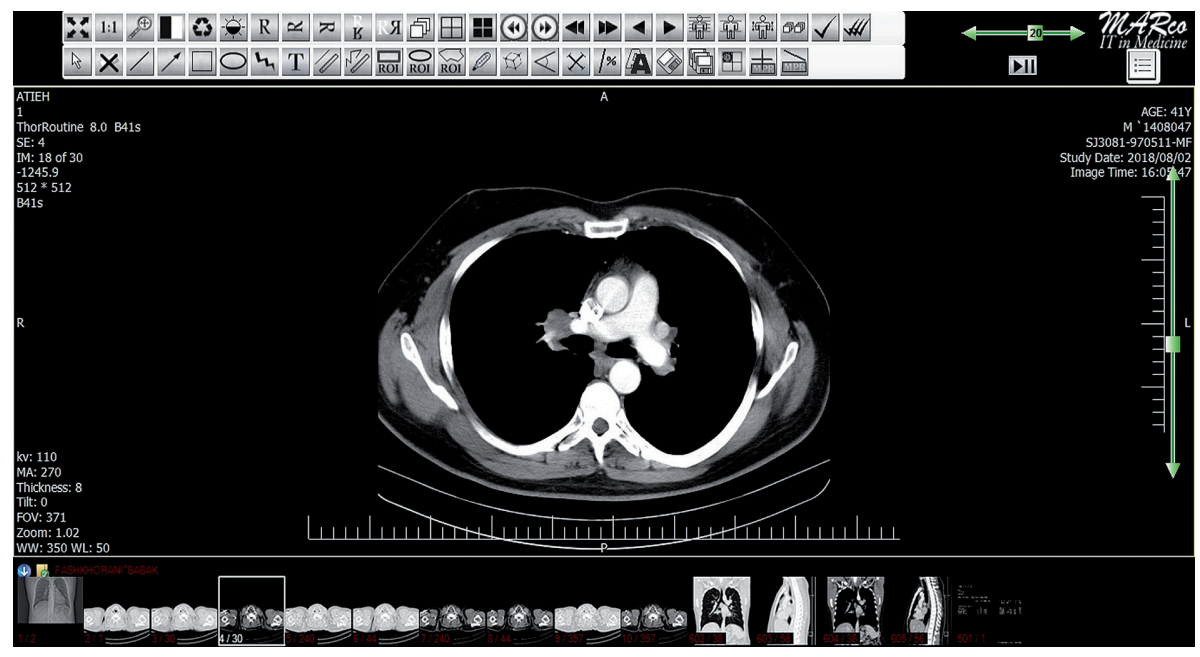

Figure 3. Hilar lymph nodes in axial computed tomography

and anterior mediastinoscopy are used [2]. But all these methods for staging are mainly invasive and would result in high costs $[3,4]$. However, the positron emission tomography (PET) scan may develop high applicability for staging of metastatic lung cancer with lymph node involvement [5], but it is less frequently used due to high costs [6]. Endoscopic ultrasound-guided fine needle aspiration (FNA) was also introduced; the main limitation for this method is the impossibility for airway assessment [7-10].

In this era some studies have reported highest applicability in cases using endobronchial ultrasonography (EBUS) with high accuracy in false negative CT scan [11-14]. However, there are some controversies about the role of the EBUS (Figures 1 and 2) versus spiral chest CT scan (Figures 3 and 4) to differentiate between infiltrated and none-infiltrated lymph nodes.

Hence, for improvement in diagnostic approaches, the purpose in this study was to determine the diagnostic accuracy of endobronchial ultrasound and spiral chest CT scan in the prediction of infiltrating and non-infiltrating lymph nodes in patients undergoing endobronchial ultrasound.

\section{Material and methods}

In this observational prospective study, 40 consecutive patients with infiltrating and non-infiltrating lymph nodes in Masih-Daneshvari Hospital, Tehran, Iran in 2017 and 2018, who had positive pathology results by biopsy or EBUS but with non-diagnostic spiral chest CT scan, were enrolled. The exclusion criterion was incomplete data.

The study was approved by the local Ethical Committee. Data were collected by existing medical documents and checklist among understudy patients. Also, the pathological findings including benign and malignant status were recorded in the patients. The size and location of the lymph nodes were also determined.

Data analysis was done with SPSS software (Version 25.0), and mean plus standard deviation were calculated for numerical variables, and the frequency and percentage were reported for categorical factors. Kappa test was used to determine the congruence, and the sensitivity, specificity, and accuracy was calculated. $P$ values less than 0.05 were considered statistically significant. 


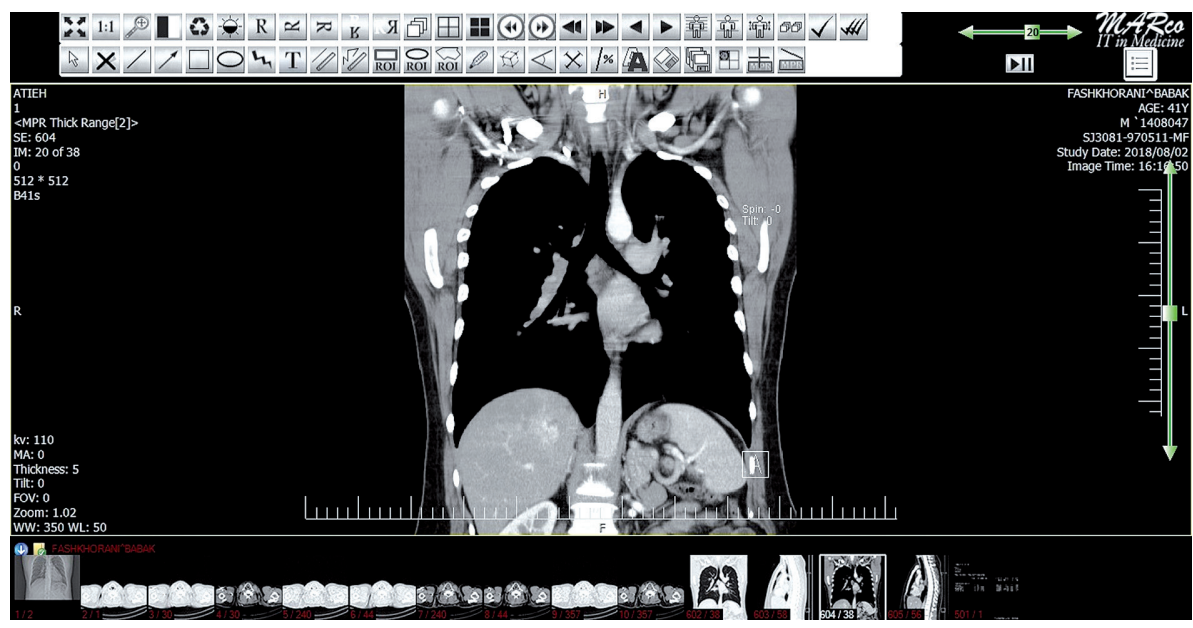

Figure 4. Hilar and subcarinal lymph nodes in coronal computed tomography

\section{Results}

The patient's age was under 50 years in 17 cases $(42.5 \%)$. The gender was male in 15 patients $(37.5 \%)$. The CT scan results were positive in 33 patients $(82.5 \%)$, and the EBUS was positive in 35 cases (87.5\%). According to the pathological results, 31 cases $(77.5 \%)$ were benign. The mean lymph node size was $10.7 \mathrm{~mm}$ in CT and $11 \mathrm{~mm}$ in EBUS without difference between benign or malignant lesions $(p>0.05)$. Also, the total range was from 6 to $25 \mathrm{~mm}$.

The results in this study (Table 1) show that the congruence between EBUS and CT scan was $80.5 \%$ $(p=0.0001)$. The sensitivity, specificity, and accuracy for CT scan was $100 \%, 22.6 \%$, and $40 \%$, respectively (Table 2) and the sensitivity, specificity, and accuracy for EBUS was $100 \%, 16.1 \%$, and $35 \%$, respectively (Table 3 ).

The congruence did not differ according to age, and patients younger and older than 50 years had good congruence between EBUS and CT scan. Also, the congruence did not differ according to gender, and both male and female patients had good congruence between EBUS and CT scan. The congruence was good in benign cases $(p=0.0001)$, but it was not determined according to the location, malignancy, and stage of the malignancy $(p>0.05)$.

\section{Discussion}

The main utilised method to diagnose mediastinal lymphadenopathy is CT scan, but it would show low accuracy. The highest applicability point is related to EBUS. However, there are some controversies. Because CT scan is a non-invasive method, in this study the accuracy of EBUS and spiral CT scan was assessed. The congruence of methods was $80.5 \%$ and significant. The sensitivity for methods was $100 \%$ and the specificity was near to $20 \%$ with accuracy rate of 35 to $40 \%$. Age and gender subgroups both had good equal concordances. The congruence was higher in benign lesions. It is not clear in malignant lesions. Also,
Table 1. Congruence between computed tomography scan and endobronchial ultrasound results

\begin{tabular}{|c|c|c|c|c|c|}
\hline & \multicolumn{2}{|c|}{ CT } & \multirow[t]{2}{*}{ Total } \\
\hline & & & Pos & Neg & \\
\hline \multirow[t]{6}{*}{ EBUS } & \multirow[t]{3}{*}{ Pos } & Count & 33 & 2 & 35 \\
\hline & & $\%$ within EBUS & $94.3 \%$ & $5.7 \%$ & $100 \%$ \\
\hline & & $\%$ within CT & $100 \%$ & $28.6 \%$ & $87.5 \%$ \\
\hline & \multirow[t]{3}{*}{$\mathrm{Neg}$} & Count & 0 & 5 & 5 \\
\hline & & $\%$ within EBUS & 0 & $100 \%$ & $100 \%$ \\
\hline & & $\%$ within $\mathrm{CT}$ & 0 & $71.4 \%$ & $12.5 \%$ \\
\hline \multirow{3}{*}{\multicolumn{2}{|c|}{ Total }} & Count & 33 & 7 & 40 \\
\hline & & $\%$ within EBUS & $82.5 \%$ & $17.5 \%$ & $100 \%$ \\
\hline & & $\%$ within CT & $100 \%$ & $100 \%$ & $100 \%$ \\
\hline
\end{tabular}

Table 2. Computed tomography scan sensitivity, specificity, and accuracy

\begin{tabular}{|c|c|c|c|c|c|}
\hline & \multicolumn{2}{|c|}{ Pathological result } & \multirow[t]{2}{*}{ Total } \\
\hline & & & Benign & Malignant & \\
\hline \multirow[t]{8}{*}{ EBUS } & \multirow[t]{4}{*}{ Pos } & Count & 24 & 9 & 33 \\
\hline & & $\%$ within CT & $72.7 \%$ & $27.3 \%$ & $100 \%$ \\
\hline & & $\%$ within & $77.4 \%$ & $100 \%$ & $82.5 \%$ \\
\hline & & Pathological result & & & \\
\hline & \multirow[t]{4}{*}{ Neg } & Count & 7 & 0 & 7 \\
\hline & & $\%$ within CT & $100 \%$ & 0 & $100 \%$ \\
\hline & & $\%$ within & $22.6 \%$ & 0 & $17.5 \%$ \\
\hline & & Pathological result & & & \\
\hline \multirow{4}{*}{\multicolumn{2}{|c|}{ Total }} & Count & 31 & 9 & 40 \\
\hline & & $\%$ within CT & $77.5 \%$ & $22.5 \%$ & $100 \%$ \\
\hline & & $\%$ within & $100 \%$ & $100 \%$ & $100 \%$ \\
\hline & & Pathological result & & & \\
\hline
\end{tabular}

the location and stage effects were impossible to determine. Udoji et al. [15] reported that lymph nodes had larg- 
Table 3. Endobronchial ultrasound sensitivity, specificity, and accuracy

\begin{tabular}{|c|c|c|c|c|c|}
\hline & \multicolumn{2}{|c|}{ Pathological result } & \multirow[t]{2}{*}{ Total } \\
\hline & & & Benign & Malignant & \\
\hline \multirow[t]{8}{*}{ EBUS } & \multirow[t]{4}{*}{ Pos } & Count & 26 & 9 & 35 \\
\hline & & $\%$ within EBUS & $74.3 \%$ & $27.5 \%$ & $100 \%$ \\
\hline & & $\%$ within & $83.9 \%$ & $100 \%$ & $87.5 \%$ \\
\hline & & Pathological result & & & \\
\hline & \multirow[t]{4}{*}{ Neg } & Count & 5 & 0 & 5 \\
\hline & & $\%$ within EBUS & $100 \%$ & 0 & $100 \%$ \\
\hline & & $\%$ within & $16.1 \%$ & 0 & $12.5 \%$ \\
\hline & & Pathological result & & & \\
\hline \multirow{4}{*}{\multicolumn{2}{|c|}{ Total }} & Count & 31 & 9 & 40 \\
\hline & & $\%$ within EBUS & $77.5 \%$ & $22.5 \%$ & $100 \%$ \\
\hline & & $\%$ within & $100 \%$ & $100 \%$ & $100 \%$ \\
\hline & & Pathological result & & & \\
\hline
\end{tabular}

er size in EBUS versus CT scan assessment. In our study, despite a lack of difference between benign and malignant lesions, it was larger in EBUS evaluation.

Helmy et al. [16] assessed 11 patients who were suspected of having lymph node malignancy without CT scan positive results. The malignancy was reported in $54.4 \%$ of cases under bronchoscopy, $18.2 \%$ under EBUS-TBNA, and 27.3\% under CT-guided biopsy. From 11 cases with negative CT scan the EBUS was positive in four patients [16]. In our study the malignancy rate was lower, but similarly EBUS had good applicability.

Nakajima et al. [17] showed that among 106 patients with metastatic lung cancer the sensitivity and specificity were $92 \%$ and $100 \%$, respectively. Their sensitivity and specificity were lower and higher than ours, respectively. Memoli et al. [18] reported malignancy in lymph nodes in $22.5 \%$ of cases and reported good diagnostic applicability for both CT scan and EBUS method as well as our study.

Jhun et al. [19] reported sensitivity and specificity of 92\% and 99\%, respectively, for EBUS, which was related to lymph node location. However, in our study, due to the small number of hilar samples, a comparison was impossible. Lee et al. [20] assessed 247 patients with hilar and mediastinal lymphadenopathy and reported that older age and pathological results were related to higher false negative rates. In our study the benign status had some effects on congruence between methods, but there was no difference for age.

According to the obtained results it may be concluded that CT scan and EBUS results have good congruence and high sensitivity to differentiate infiltrating and non-infiltrating lymph nodes. Hence, these methods are useful for screening methods, but due to their low specificity and accuracy their use for a confirmative approach is not beneficial. However, regarding its accessibility and less invasive nature, the use of chest CT scan is more rational and is recommended in these patients. However further studies with larger sample sizes and controlling for confounding factors would develop more definite results.

\section{Disclosure}

The authors declare no conflicts of interest.

\section{References}

1. Toloza EM, Harpole L, Detterbeck F, McCrory DC. Invasive staging of non-small cell lung cancer: a review of the current evidence. Chest 2003; 123: 157-166.

2. Hoffmann H. Invasive staging of lung cancer by mediastinoscopy and video-assisted thoracoscopy. Lung Cancer 2001; 34: 3-5.

3. Luke WP, Pearson FG, Todd TR, et al. Prospective evaluation of mediastinoscopy for assessment of carcinoma of the lung. J Thorac Cardiovasc Surg 1986; 91: 53-56.

4. Coughlin M, Deslauriers J, Beaulieu M, et al. Role of mediastinoscopy in pretreatment staging of patients with primary lung cancer. Ann Thorac Surg 1985; 40: 556-560.

5. Toloza EM, Harpole L, McCrory DC. Noninvasive staging of nonsmall cell lung cancer: a review of the current evidence. Chest 2003 123 Suppl 1: 137-146.

6. Brim O, Kappetein AP, Stijnen T, Bogers AJ. Meta-analysis of positron emission tomographic and computed tomographic imaging in detecting mediastinal lymph node metastases in nonsmall cell lung cancer. Ann Thorac Surg 2005; 79: 375-382.
7. Jacobson BC, Hirota WK, Goldstein JL, et al. The role of EUS for evaluation of mediastinal adenopathy. Gastrointest Endoscopy 2003; 58: $819-821$

8. Wallace MB, Woodward TA, Raimondo M. Endoscopic ultrasound and staging of non-small cell lung cancer. Gastrointest Endosc Clin N Am 2005; 5: 157-167.

9. Annema JT, Versteegh MI, Veselic M, et al. Endoscopic ultrasound-guided fine-needle aspiration in the diagnosis and staging of lung cancer and its impact on surgical staging. J Clin Oncol 2005; 23: 8357-8361.

10. Annema JT, Versteegh MI, Veselic M, et al. Endoscopic ultrasound added to mediastinoscopy for preoperative staging of patients with lung cancer. JAMA 2005; 294: 931-936.

11. Krasnik M, Vilmann P, Larsen SS, Jacobsen GK. Preliminary experience with a new method of endoscopic transbronchial real time ultrasound guided biopsy for diagnosis of mediastinal and hilar lesions. Thorax 2003; 58: 1083-1088. 
12. Yasufuku K, Chiyo M, Sekine Y, et al. Real-time endobronchial ultrasound-guided transbronchial needle aspiration of mediastinal and hilar lymph nodes. Chest 2004; 126: 122-128.

13. Rintoul RC, Skwarski KM, Murchison JT, et al. Endobronchial and endoscopic ultrasound-guided real-time fine-needle aspiration for mediastinal staging. Eur Respir J 2005; 25: 416-421

14. Okamoto H, Watanabe K, Nagatomo A, et al. Endobronchial ultrasonography for mediastinal and hilar lymph node metastases of lung cancer. Chest 2002; 121: 1498-1506.

15. Udoji TN, Phillips GS, Berkowitz EA, et al. Mediastinal and hilar lymph node measurements. comparison of multidetector-row computed tomography and endobronchial ultrasound. Ann Am Thorac Soc 2015; 12: 914-920.

16. Helmy NA, Kamel KM, Naglaa BA. Endobronchial ultrasonography for lung cancer staging in negative mediastinum on computed tomography. Egypt J Chest Dis Tuberc 2013; 62: 475-479.
17. Nakajima T, Yasufuku K, Iyoda A, et al. The evaluation of lymph node metastasis by endobronchial ultrasound-guided transbronchial needle aspiration: crucial for selection of surgical candidates with metastatic lung tumors. J Thorac Cardiovasc Surg 2007; 134: 1485-1490.

18. Wang Memoli JS, El-Bayoumi E, Pastis NJ, et al. Using endobronchial ultrasound features to predict lymph node metastasis in patients with lung cancer. Chest 2011; 140: 1550-1556.

19. Jhun BW, Park HY, Jeon K, et al. Nodal stations and diagnostic performances of endobronchial ultrasound-guided transbronchial needle aspiration in patients with non-small cell lung cancer. J Korean Med Sci 2012; 27: 46-51.

20. Lee J, Kim YK, Seo YY, et al. Clinical characteristics of false-positive lymph node on chest CT or PET-CT confirmed by endobronchial ultrasound-guided transbronchial needle aspiration in lung cancer. Tuberc Respir Dis (Seoul) 2018; 81: 339-346. 